\title{
Narrative review of the emerging role of molecular biomarkers in guiding the definitive management of unresectable non-small cell lung cancer
}

\author{
Eric K. Singhi, Carl M. Gay \\ Department of Thoracic/Head and Neck Medical Oncology, The University of Texas, MD Anderson Cancer Center, Houston, Texas, USA \\ Contributions: (I) Conception and design: All authors; (II) Administrative support: All authors; (III) Provision of study materials or patients: None; \\ (IV) Collection and assembly of data: All authors; (V) Data analysis and interpretation: All authors; (VI) Manuscript writing: All authors; (VII) Final \\ approval of manuscript: All authors. \\ Correspondence to: Carl M. Gay, MD, PhD. Department of Thoracic/Head and Neck Medical Oncology, The University of Texas, MD Anderson \\ Cancer Center, Houston, Texas, USA. Email: cgay@mdanderson.org.
}

\begin{abstract}
The addition of PD-L1 targeting consolidation therapy to previously standard of care concurrent chemoradiation for locally advanced, unresectable non-small cell lung cancer resulted in dramatic improvements in clinical outcomes. However, in contrast to patients with metastatic disease, the application of immunotherapies is not currently guided by molecular characteristics of patient tumors. Furthermore, despite increasing awareness of predictive and/or prognostic genomic alterations in patients with locally advanced disease, the utility of targeted therapies, such as those aimed at alterations in EGFR or ALK, remains unclear in this subset of patients. As a result, patients with unresectable, locally advanced non-small cell lung cancer are treated uniformly according to histology, regardless of other molecular features despite the potential for treatment-associated risks without a clear benefit. Here, we first discuss the advantages of utilizing molecular biomarkers to guide treatment of non-small cell lung cancer based on treatment outcomes in the metastatic setting. Next, we review preclinical and retrospective clinical data that supports potential further personalization of these treatment strategies in earlier stages of disease. Finally, we discuss some of the ongoing clinical trials attempting to address these hypotheses prospectively.
\end{abstract}

Keywords: Immunotherapy; targeted therapy; unresectable non-small cell lung cancer (unresectable NSCLC)

Submitted Feb 24, 2020. Accepted for publication Oct 15, 2020.

doi: $10.21037 /$ tlcr-20-330

View this article at: http://dx.doi.org/10.21037/tlcr-20-330

\section{Introduction}

Eighty-five percent of all lung cancer diagnoses are non-small cell lung cancer (NSCLC), with approximately one-third of these cases presenting as locally advanced disease (1). The results of the PACIFIC trial (NCT02125461) redefined the standard of care for unresectable, locally advanced NSCLC by demonstrating that consolidation with durvalumab following concurrent chemotherapy and radiation therapy (CCRT) resulted in improved progression-free survival (PFS) and overall survival (OS) $(1,2)$. In fact, the PACIFIC trial demonstrated a 12 -month PFS of $56 \%$ with durvalumab as compared to $35 \%$ with placebo. Although a significant improvement, $44 \%$ of patients still progressed or died within the first year of treatment. This represents an unmet need for novel treatment strategies in these potentially curable patients.

Over the years, the identification of molecular biomarkers has offered both predictive and prognostic insight into the outcomes of patients with metastatic NSCLC. Subsequent utilization of these biomarkers has helped tailor therapy for patients and maximize the therapeutic benefit derived from a particular treatment regimen. As a result, it is now essential to perform biomarker testing in the metastatic setting to identify actionable genetic variants. Targeted agents have only increased with time and have proven to effectively decrease tumor burden and improve the quality of life for many patients. At present, FDA approved targeted therapies in the metastatic setting are available for 
Table 1 FDA approved targeted therapies for metastatic NSCLC as of 10/2020

\begin{tabular}{ll}
\hline Target & FDA approved drugs \\
\hline ALK rearrangement & Alectinib, brigatinib, ceritinib, crizotinib, lorlatinib \\
BRAFV600E & Dabrafenib, dabrafenib/trametinib, vemurafenib \\
EGFR sensitizing mutation & Afatinib, dacomitinib, erlotinib, gefitinib, osimertinib \\
$M E T$ exon 14 skipping mutation & Capmatinib, crizotinib \\
NTRK gene fusion & Entrectinib, larotrectinib \\
$R E T$ rearrangement & Cabozantinib, pralsetinib, selpercatinib, vandetanib \\
$R O S 1$ rearrangement & Ceritinib, crizotinib, entrectinib, lorlatinib \\
\hline
\end{tabular}

NSCLC, non-small cell lung cancer.

molecular alterations in the following genes: EGFR, $A L K$, ROS1, NTRK1, BRAF, RET, and MET (Table 1). Promising data evaluating the safety and efficacy of a KRAS G12C inhibitor was also recently published in the literature (3). It is also important to note that upfront PD-L1 expression testing should be performed in all patients with metastatic NSCLC before first-line therapy is administered.

Although our care for patients with metastatic NSCLC now routinely incorporates a personalized approach to treatment based on molecular profiling of the tumor, a similar treatment approach has not been the standard practice for our patients with locally advanced disease. In the future, molecular profiling may no longer be restricted to just the metastatic setting, as its role in earlier stage disease is becoming more relevant. As an example, the recently published results of the ADAURA trial demonstrated a potential role for adjuvant osimertinib in patients with stage IB to IIIA EGFR-mutant NSCLC following previous surgical resection (4). Results from the trial demonstrated a very significant disease-free survival benefit for adjuvant osimertinib and may prove to change practice in the treatment of this patient population. Longer follow-up and data about sites of disease recurrence, however, are still needed to determine if this benefit will ultimately translate into improvements in OS and quality of life.

Currently, the standard of care management of unresectable locally advanced NSCLC involves the administration of definitive CCRT followed by durvalumab as consolidation immunotherapy (1). Notably, durvalumab is not recommended for patients who have had definitive surgical resection. NCCN guidelines recommend that if patients do plan to receive durvalumab but have not received full-dose chemotherapy concurrently with radiation therapy, then consolidation chemotherapy should be avoided given the increased risk of pneumonitis if patients are also to receive durvalumab. Alternatively, if patients will not be receiving durvalumab because they are ineligible for immunotherapy, consolidation chemotherapy may be considered after CCRT has been completed. The following review article will explore the use of various biomarkers to personalize this standard approach for the treatment of patients with stage III unresectable NSCLC. We present the following article in accordance with the Narrative review checklist (available at http://dx.doi.org/10.21037/tlcr-20-330).

\section{PACIFIC trial}

The PACIFIC trial was a pivotal study that evaluated the use of immune checkpoint blockade in patients with locally advanced NSCLC (1). In the study, 713 patients who had been treated with at least two cycles of platinum-based chemotherapy with radiation and were without evidence of disease progression were randomized in a 2:1 ratio to receive durvalumab at $10 \mathrm{mg} / \mathrm{kg}$ every 2 weeks for up to 1 year or to receive placebo. The primary endpoints of the study were defined as PFS and OS. At a median follow-up of 14.5 months, the PFS in the intent-to-treat population was significantly improved at 16.8 months in the durvalumab consolidation arm as compared to 5.6 months in the placebo arm $(\mathrm{HR}=0.52, \mathrm{P}<0.001)$. In 2018, updated results from the PACIFIC trial demonstrated that after a median follow-up of 25.2 months, OS in the intent-to-treat population was significantly improved in patients treated with durvalumab as compared to treatment with placebo ( $\mathrm{HR}=0.68, \mathrm{P}=0.0025)(2)$.

Results of the PACIFIC trial were indeed practicechanging, as it significantly transformed the standard of care for locally advanced, unresectable NSCLC. Prior to the PACIFIC trial, the standard of care in this setting had been a combination of at least two cycles of platinum-based chemotherapy along with concurrent radiation therapy to visible disease and the mediastinum for a total of 6-7 weeks 
of therapy with a cure rate of $20-25 \%$. Now, as a direct result of the PACIFIC trial, all immunotherapy-eligible patients with locally advanced NSCLC who have completed definitive CCRT should receive durvalumab for 1 year given its overall tolerability and striking PFS and OS benefit.

In an attempt to better understand which patients benefit most from durvalumab consolidation after CCRT, a prespecified analysis of outcomes with respect to PDL1 expression was conducted with a $25 \%$ cutoff point. PD-L1 testing was performed using the Ventana SP263 immunohistochemical assay on patients' archival tumor tissue obtained prior to CCRT. The subgroup analysis of OS benefit showed that with a PD-L1 expression of $25 \%$ as a defined cutoff point, treatment with durvalumab was associated with an improvement in PFS regardless of the tumor's PD-L1 expression, with a $\mathrm{HR}=0.59$ for PDL1 expression level $<25 \%$ and HR 0.41 for a PD-L1 expression level of $\geq 25 \%$.

As part of an unplanned post hoc analysis, patients with PD-L1 expression of less than $1 \%$ demonstrated a trend towards failing to achieve an OS benefit from durvalumab consolidation. Specifically, patients with PD-L $1 \geq 1 \%$ had a HR $=0.53$ (95\% CI: 0.36-0.77) favoring the durvalumab arm, as compared to patients with PD-L1 $<1 \%$ had a HR $=1.36$ (95\% CI: 0.79-2.34) favoring treatment with placebo. Although these results are hypothesis generating, it is important to keep in mind that the PACIFIC trial was not designed to evaluate the efficacy of durvalumab consolidation with regards to PD-L1 expression and it did not prospectively stratify patients at time of randomization by PD-L1 status. Additionally, the trial studied a limited number of patients with tumor PD-L1 expression of $<1 \%$ $(\mathrm{n}=148)$. Furthermore, not all patients in the PACIFIC trial provided tumor tissue for assessment of PD-L1 expression; whereas for those that did, PD-L1 testing was performed on tissue obtained prior to CCRT, which may not be entirely representative of the tumor microenvironment changes and changes in PD-L1 expression that occur secondary to CCRT $(5,6)$. Nevertheless, these results raise concerns about the use of the PACIFIC regimen irrespective of PDL1 expression and could have potential implications for patient selection in the future.

Concerning patient enrollment, it is important to note that the PACIFIC trial did not exclude patients on the basis of next generation sequencing, including those with EGFR mutations or ALK translocations. Although the number of patients harboring these driver mutations was limited, subgroup analysis of patients based on their EGFR mutation status showed that while EGFR-mutated patients still favored treatment with durvalumab, the trend was towards a more modest benefit. Specifically, the PACIFIC trial included $43 / 713$ patients (6\%) with EGFR-mutated disease. Subgroup analysis of PFS with regards to EGFR mutation status revealed that patients with a known EGFR mutation had a HR $=0.76$ (95\% CI: $0.35-1.64$ ) as compared to patients with a wild-type EGFR status who had a HR $=0.47$ (95\% CI: 0.36-0.60). Clinically, like the aforementioned results with PD-L1, these results are difficult to interpret, but are provocative considering the role that driver mutations might play in the treatment of locally advanced NSCLC.

For now, the PACIFIC regimen remains the standard of care for treatment of our unresectable locally advanced NSCLC patients, regardless of genomic or proteomic biomarkers. We are still awaiting further studies to better determine more personalized treatment options for patients in this setting based on molecular profiling. Additionally, the decision to continue with durvalumab consolidation beyond 1 year remains challenging and is ultimately a discussion to be had between patients and their providers.

\section{KRAS-mutant NSCLC}

Beyond the PACIFIC trial, additional studies have investigated how best to utilize known biomarkers when identifying treatment options for patients with unresectable locally advanced NSCLC. One such biomarker is KRAS mutation status. Clinically, KRAS is the most commonly mutated oncogene in NSCLC. There are multiple KRAS-mutant subtypes of NSCLC that are largely defined by co-mutations in TP53 and LKB1 (STK11) (7). LKB1/STK11 is a tumor suppressor gene involved in the AMPK-mTOR pathway that directly impacts cell growth and metabolism. Previous studies have shown that KRAS-mutated tumors harboring co-mutations in STK11 are often resistant to conventional treatment modalities consisting of chemotherapy and radiation (8-11). Skoulidis et al. additionally demonstrated that LKB1 (STK11) promotes resistance to immune checkpoint blockade in KRAS-mutant lung adenocarcinoma murine models (12). Specifically, the investigators showed that alterations in LKB1 were associated with a lack of PD-L1 expression in tumor cells across multiple cohorts, despite an intermediate or even high tumor mutational burden (TMB); additionally, there were lower concentrations of infiltrating $\mathrm{CD} 8^{+}$cytotoxic $\mathrm{T}$ lymphocytes in both human and murine LKB1 deficient tumors. The mechanism of poor PD-L1 expression and $\mathrm{T}$ cell infiltration is thought to be secondary to epigenetic silencing of the stimulator of interferon genes (STING) 
pathway, which is known to have an essential role in innate immunity. Loss of LKB1/STK11 results in suppression of the STING pathway, resulting in decreased PD-L1 expression and T-cell recruitment (13). To improve upon treatment response rates, Wang et al. explored how best to sensitize KRAS-STK11 mutant tumors to radiation, and showed that this specific subtype of NSCLC is sensitive to a combination of trametinib (MEK 1/2 inhibitor) and radiation (14). The leading hypothesis to best explain the radiosensitization mechanism observed is that this combination acts on two tumor suppressor pathways at the same time. Specifically, the combination works to stabilize the $\mathrm{p} 53$ protein and activates the AMP-activated protein kinase (AMPK) depending on LKB1 to ultimately induce senescence on cells. It is important to note that combination therapy was not effective in KRASmutated tumors that lack STK11 mutations, including those with co-mutated TP53.

Although no therapies targeting KRAS mutations in NSCLC have been formally approved by the FDA to date, recently published data of a phase 1 trial of sotorasib showed promising clinical benefit in patients with advanced solid tumors harboring the KRAS G12C mutation. In fact, $32 \%$ of the patients in the subgroup with NSCLC had a confirmed objective response to therapy, despite being heavily pre-treated (3). Future research should consider how best to integrate KRAS-targeting therapy into the treatment paradigm for earlier stages of disease.

\section{EGFR- and ALK-mutant NSCLC}

Pre-dating the PACIFIC trial, Tanaka et al. demonstrated that EGFR mutation status offers predictive insight on the response to definitive CCRT in unresectable stage III NSCLC (15). This is an important concept to explore as retrospective studies suggest that up to $30 \%$ of patients in some populations with locally advanced NSCLC harbor an EGFR mutation $(16,17)$. Tanaka $e t$ al. performed a retrospective analysis and found that PFS in patients treated with CCRT was significantly shorter in those with EGFR-mutated NSCLC as compared to their wild-type counterparts [median PFS (mPFS) $9.8 v s$. 16.5 months, $\mathrm{P}=0.041]$ (15). The investigators also noted that EGFR-mutated NSCLC was more likely to develop distant metastases after CCRT as compared to wild-type EGFR lung adenocarcinomas, with the brain being the most common site in EGFR-mutant patients (35\%). Interestingly, locoregional recurrence was lower in EGFR-mutant patients than for their wild-type counterparts ( $14 \%$ vs. $35 \%, \mathrm{P}=0.027)$. The higher frequency of distant metastases, particularly in the brain, suggests an intrinsic brain tropism of the EGFR-mutant tumors; one can hypothesize that CCRT with or without durvalumab consolidation is likely not penetrating the bloodbrain barrier as effectively.

To better understand the impact of EGFR and $A L K$ mutations on definitive CCRT, Nakamura et al. evaluated 173 patients with lung adenocarcinoma, 34 with EGFRmutated disease and 13 with EML4-ALK translocation (18). The investigators again found that in the EGFR-mutant group, there was a significantly lower rate of in-field failure $(\mathrm{P}=0.027)$, however, there was a higher rate of out-offield failure $(\mathrm{P}=0.029)$ as compared to the wild-type $E G F R$ adenocarcinoma group. This suggests that EGFR-mutated adenocarcinoma may be more chemoradiosensitive. There was no significant difference in rates of in-field or out of field failure between the $A L K$-translocated and wild-type $A L K$ groups, albeit the numbers were small. Interestingly, the 3 -year OS rate was significantly higher for EGFR-mutant patients than their wild-type counterparts ( $75 \%$ vs. $46 \% ; \mathrm{P}=0.002)$; this is likely because patients in the EGFR-mutant group received tyrosine kinase inhibitors (TKIs) after recurrence; while the wild-type group went on to receive salvage chemotherapy. Limitations of this study should be acknowledged, including its retrospective nature and small sample size. Nevertheless, this study hints that molecular profiling of the tumor may offer insight on the likelihood of recurrence after definitive CCRT in our locally advanced unresectable NSCLC patients. It also raises the question of whether similar strategies may be viable for patients harboring other targetable mutations (i.e., MET, RET, ROS1, BRAFV600E, etc.)

\section{KEAP1- or NRF2-mutant squamous cell carcinomas (SCC)}

Focusing specifically on the treatment of SCC of the lung, a subtype of NSCLC, studies have been conducted to improve upon the resistance to radiation therapy that KEAP 1 or $N R F 2$ mutations induce, as previous investigation has shown increased radiotherapy resistance in tumors harboring this specific subset of mutations. The KEAP1-NRF2 pathway plays a protective role against oxidative stress. Specifically, NRF2 is a transcription factor that is normally bound by the adapter protein KEAP1. When oxidative stress is present, NRF2 is released from KEAP1 and subsequently translocates into the nucleus to induce transcription of genes that defend against reactive oxygen species (ROS). Mutations in KEAP1 or NRF2 are present in more than one third of patients with SCC of the lung. Previous studies have shown that the KEAP1 or NRF2 pathway and production of ROS affect the 
Table 2 Selected biomarker-driven trials for patients with locally advanced NSCLC

\begin{tabular}{|c|c|c|c|c|c|}
\hline Trial & Phase & $\begin{array}{l}\text { Estimated number } \\
\text { of patients }\end{array}$ & $\begin{array}{l}\text { Biomarker for } \\
\text { eligibility }\end{array}$ & Radiation type & Systemic treatment \\
\hline WJOG6911L & 2 & 27 & EGFR & Conventional & Gefitinib \\
\hline NCT01391260 & 2 & 30 & EGFR & Conventional & Gefitinib \\
\hline RT0901 & 2 & 75 & EGFR & Conventional & Erlotinib \\
\hline SPRINT & 2 & 63 & PD-L1 & Conventional & $\begin{array}{l}\text { Pembrolizumab (PD-L1 } 250 \% \text { ) or } \\
\text { conventional CCRT (PD-L1 <50\%) }\end{array}$ \\
\hline
\end{tabular}

CCRT, concurrent chemotherapy and radiation therapy.

development and growth of lung cancer; additional studies have also demonstrated the effects of KEAP1 or NRF2 mutations on treatment resistance, with one hypothesis being that high levels of ROS can lead to suppression of $\mathrm{CD} 8^{+} \mathrm{T}$ cells, resulting in suppression of the overall immune system and ultimately treatment resistance (19). Jeong et al. developed a murine model of SCC of the lung and demonstrated that deletion of KEAP1 or Trp53 (TP53 in humans) promotes resistance to radiation therapy in this specific subset of patients (20). Specifically, the investigators found that KEAP1/NRF2 mutation status is predictive of the rate of local progression after radiotherapy in SCC of the lung. Their results are clinically meaningful and suggest that patients with KEAP1 or $N R F 2$ alterations might benefit from radiation dose escalation, an alternate modality of local therapy, or inhibition of NRF2 targets to allow for radiosensitization. Prospective studies are needed to further investigate a more personalized treatment approach for this subset of patients.

\section{Emerging treatment strategies}

Additional studies using various biomarker-based strategies are already underway with the hope to redefine treatment of our patients with locally advanced NSCLC (Table 2 ). With the reported differences in response to CCRT based on EGFR and $A L K$ mutation status, several trials are investigating the role of TKIs in the treatment of locally advanced NSCLC, both in the consolidation phase and in lieu of platinum-based chemotherapy. As an example, Akamatsu et al. are enrolling 27 patients in the single arm phase II study WJOG6911L, which will investigate the efficacy and safety of concurrent gefitinib and thoracic radiotherapy in locally advanced EGFR-mutant NSCLC (21). The rationale for the trial is sound as the use of EGFR-TKIs as compared to platinum-doublet chemotherapy has a significantly improved objective response rate (ORR) in the treatment of metastatic NSCLC; preclinical study also suggests that gefitinib has a radio-sensitizing effect (22). The primary endpoint is PFS at 2 years with key secondary endpoints to include ORR, PFS, OS, and safety. Other noteworthy studies among several that will specifically focus on patients with EGFR-mutant locally advanced NSCLC include the phase II LOGIK0902/OLCSG0905 intergroup study evaluating the use of induction gefitinib monotherapy followed by CCRT, phase II NCT01391260 studying concurrent gefitinib and thoracic radiation therapy, and the phase II RT0901 trial evaluating concurrent erlotinib and thoracic radiation therapy.

Further attempts to move away from platinum-based chemotherapy using a biomarker directed approach include the NRG-LU004 phase I trial. Specifically, the NRG-LU004 trial will evaluate the safety of conventionally fractionated or accelerated hypofractionated radiotherapy in combination with durvalumab (in lieu of chemotherapy) in locally advanced NSCLC patients with a high PD-L1 expression of $50 \%$ or greater. Patients will be randomized to receive radiation therapy as 60 Gy in 30 fractions or 60 Gy in 15 fractions. Similarly, the phase II SPRINT trial (NCT03523702) will evaluate replacing concurrent chemotherapy with immunotherapy, albeit with pembrolizumab, in patients with a high ( $\geq 50 \%$ ) PD-L1 expression (23).

Finally, it is also important to think about how we can use biomarkers in the post-treatment setting, not only to evaluate treatment response, but to also tailor subsequent treatment strategies. Moding et al. explored how the use of circulating tumor DNA (ctDNA) molecular residual disease (MRD) may improve outcomes for our patients with locally advanced NSCLC (24). The investigators performed deep sequencing ctDNA analysis on 218 samples obtained from 65 patients receiving CCRT for locally advanced NSCLC; 28 of these patients were receiving consolidation 


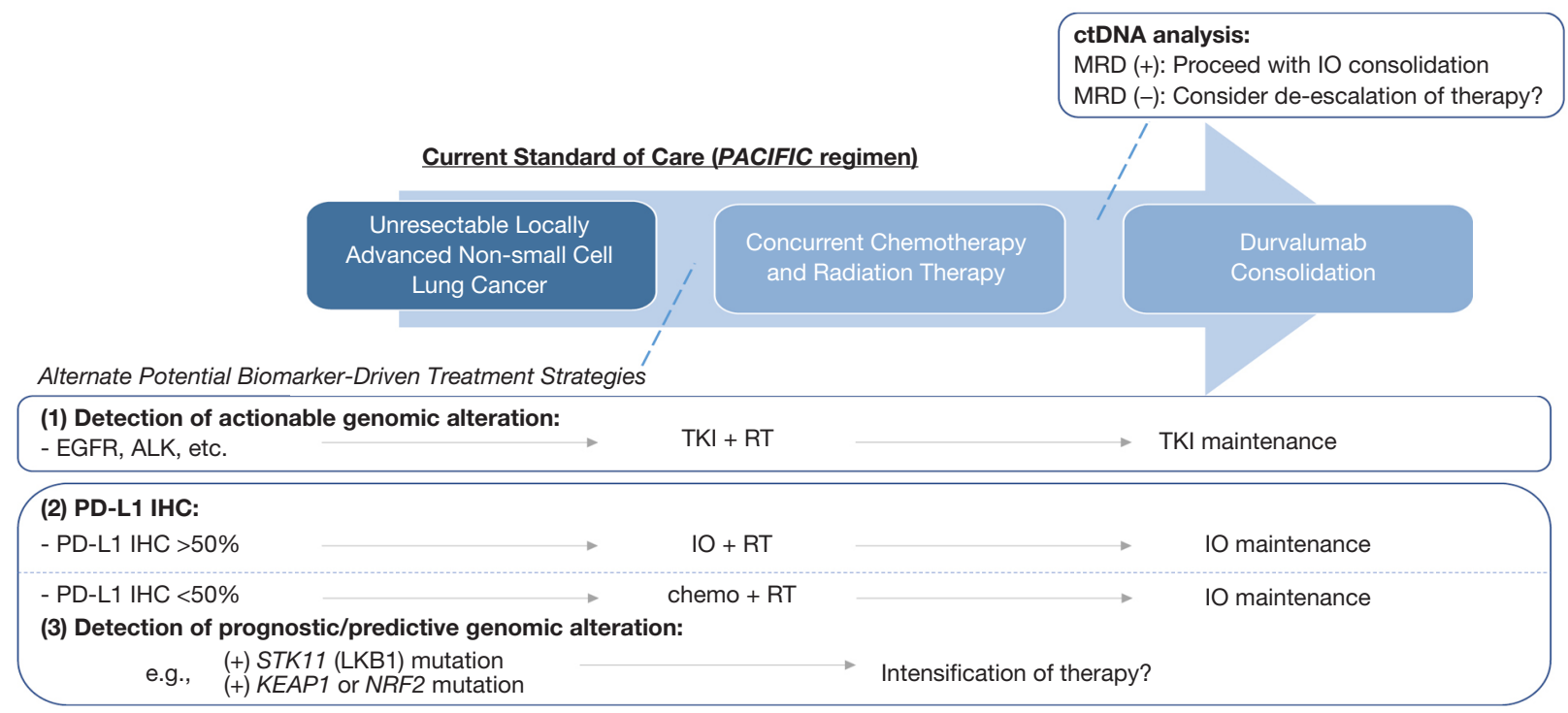

Figure 1 Potential biomarker-based treatment strategies for unresectable locally advanced NSCLC. The current standard of care for treatment of patients with unresectable locally advanced non-small cell lung cancer (NSCLC) per results of the PACIFIC trial is highlighted in the blue arrow above, and demonstrates definitive concurrent chemotherapy and radiation therapy (CCRT) followed by durvalumab consolidation, irrespective of PD-L1 expression. Alternatively, the figure above depicts potential biomarker-driven treatment strategies to consider in lieu of the current standard of care by evaluating for actionable genomic alterations, PD-L1 IHC expression, and/or other prognostic/predictive genomic alterations at time of diagnosis. Circulating tumor DNA (ctDNA) analysis for evidence of minimal residual disease (MRD) could also play an important role in treatment decision making after completion of definitive CCRT to either proceed with durvalumab consolidation or consider a de-escalation of therapy. IHC, immunohistochemistry; TKI, tyrosine kinase inhibitor; RT, radiotherapy; IO, immunotherapy.

durvalumab. The authors found that patients with undetectable ctDNA after CCRT had favorable outcomes, irrespective of subsequent consolidation immunotherapy. On the other hand, patients with MRD after CCRT had better outcomes with consolidation immunotherapy as compared to those who did not. Their work suggests that ctDNA MRD may serve as a post-treatment biomarker to assess response to treatment and potentially help us decide which patients should receive consolidation immunotherapy as a preliminary attempt to de-escalate treatment

\section{Conclusions}

The PACIFIC study was the first trial to demonstrate a survival benefit with the use of immunotherapy in earlierstage NSCLC. As a result, consolidation immunotherapy after CCRT has become the standard of care for our patients with unresectable locally advanced NSCLC, regardless of PD-L1 expression. Multiple clinical trials are being developed or are already underway to evaluate the addition of upfront immunotherapy to CCRT (i.e., DETERRED, PACIFIC2, KEYNOTE-799, NICOLAS). Alternatively, there are several studies investigating the replacement of concurrent chemotherapy, either with immunotherapy (based on level of PD-L1 expression) or targeted therapies (based on tumor mutational status) (25). We acknowledge that many of the studies cited in our discussion evaluated a small sample size. Thus, there are limitations with interpretation and the ability to arrive at definitive conclusions. Nevertheless, these studies are informative as responses to targeted therapy are typically homogenous. Therefore, these studies are useful and suggest potential methods for incorporating biomarkers into the future management of patients with locally advanced unresectable NSCLC. Larger studies are still needed to better understand the evolving role of utilizing biomarkers in this setting.

As we think ahead about the future management of NSCLC, research in this field has already begun to move away from utilizing nonselective cytotoxic chemotherapy to embracing a more personalized medicine type of approach. As such, the guidelines for treatment of patients with metastatic disease have largely shifted focus to the identification of targetable driver mutations and immune checkpoints. Future research is likely to explore novel ways to incorporate a more personalized approach to treatment in earlier stages of disease (Figure 1). Future research efforts should aim to (I) identify new targetable genetic mutations/ 
potential biomarkers and (II) utilize this information to optimize our treatment approach (i.e., combination therapy $v s$. maintenance therapy with a targeted agent etc.) to improve upon treatment outcomes, while reducing treatment-associated toxicities.

\section{Acknowledgments}

Funding: None.

\section{Footnote}

Provenance and Peer Review: This article was commissioned by the Guest editors (Steven H. Lin, Xiaolong Fu and Zhengfei Zhu) for the series "New era of treatment for unresectable locally advanced non-small cell lung cancer" published in Translational Lung Cancer Research. The article has undergone external peer review.

Reporting Checklist: The authors have completed the Narrative Review Checklist. Available at http://dx.doi. org/10.21037/tlcr-20-330

Conflicts of Interest: Both authors have completed the ICMJE uniform disclosure form (available at http:// dx.doi.org/10.21037/tlcr-20-330). The series "New era of treatment for unresectable locally advanced non-small cell lung cancer" was commissioned by the editorial office without any funding or sponsorship. CG reports grants from AstraZeneca. The other author has no other conflicts of interest to declare.

Ethical Statement: The authors are accountable for all aspects of the work in ensuring that questions related to the accuracy or integrity of any part of the work are appropriately investigated and resolved.

Open Access Statement: This is an Open Access article distributed in accordance with the Creative Commons Attribution-NonCommercial-NoDerivs 4.0 International License (CC BY-NC-ND 4.0), which permits the noncommercial replication and distribution of the article with the strict proviso that no changes or edits are made and the original work is properly cited (including links to both the formal publication through the relevant DOI and the license).
See: https://creativecommons.org/licenses/by-nc-nd/4.0/.

\section{References}

1. Antonia SJ, Villegas A, Daniel D, et al. Durvalumab after chemoradiotherapy in stage III non-small-cell lung cancer. N Engl J Med 2017;377:1919-29.

2. Antonia SJ, Villegas A, Daniel D, et al. Overall survival with durvalumab after chemoradiotherapy in stage III NSCLC. N Engl J Med 2018;379:2342-50.

3. Hong DS, Fakih MG, Strickler JH, et al. KRASG12C Inhibition with Sotorasib in Advanced Solid Tumors. N Engl J Med 2020;383:1207-17.

4. Wu YL, Tsuboi M, He J, et al. Osimertinib in Resected EGFR-Mutated Non-Small-Cell Lung Cancer. N Engl J Med 2020. [Epub ahead of print]. doi:10.1056/ NEJMoa2027071.

5. Fujimoto D, Uehara K, Sato Y, et al. Alteration of PDL1 expression and its prognostic impact after concurrent chemoradiation therapy in non-small cell lung cancer patients. Sci Rep 2017;7:11373.

6. Yoneda K, Kuwata T, Kanayama M, et al. Alteration in tumoural PD-L1 expression and stromal CD8-positive tumour-infiltrating lymphocytes after concurrent chemoradiotherapy for non-small cell lung cancer. Br J Cancer 2019;121:490-6.

7. Skoulidis F, Byers LA, Diao L, et al. Co-occurring genomic alterations define major subsets of KRAS-mutant lung adenocarcinoma with distinct biology, immune profiles, and therapeutic vulnerabilities. Cancer Discov 2015;5:860-77.

8. Herter-Sprie GS, Korideck H, Christensen CL, et al. Image-guided radiotherapy platform using single nodule conditional lung cancer mouse models. Nat Commun 2014;5:5870. Erratum in: Nat Commun. $2020 \mathrm{Apr}$ 9;11(1):1835. doi: 10.1038/s41467-020-15348-z.

9. Chen Z, Cheng K, Walton Z, et al. A murine lung cancer co-clinical trial identifies genetic modifiers of therapeutic response. Nature 2012;483:613-7.

10. Wang M, Han J, Marcar L, et al. Radiation Resistance in KRASMutated Lung Cancer Is Enabled by Stem-like Properties Mediated by an OsteopontinEGFR Pathway. Cancer Res 2017;77:2018-28

11. Williams TM, Yang L, Estrada A, et al. KRAS Oncogenic Mutations Induce Intrinsic Resistance to Radiation Through Up-Regulation of DNA Repair Pathways. Int J 
Radiat Oncol 2016;96:S238.

12. Skoulidis F, Goldberg ME, Greenawalt DM, et al. STK11/LKB1 Mutations and PD-1 Inhibitor Resistance in KRAS-Mutant Lung Adenocarcinoma. Cancer Discov 2018;8:822-35.

13. Kitajima S, Ivanova E, Guo S, et al. Suppression of STING associated with LKB1 loss in KRAS-driven lung cancer. Cancer Discov 2019;9:34-45.

14. Wang Y, Li N, Jiang W, et al. Mutant LKB1 Confers Enhanced Radiosensitization in Combination with Trametinib in KRAS-Mutant Non-Small Cell Lung Cancer. Clin Cancer Res 2018;24:5744-56.

15. Tanaka K, Hida T, Oya Y, et al. EGFR mutation impact on definitive concurrent chemoradiation therapy for inoperable stage III adenocarcinoma. J Thorac Oncol 2015;10:1720-5.

16. Akamatsu H, Kaira K, Murakami H, et al. The impact of clinical outcomes according to EGFR mutation status in patients with locally advanced lung adenocarcinoma who received concurrent chemoradiotherapy. Am J Clin Oncol 2014;37:144-7.

17. Hayashi H, Okamoto I, Kimura H, et al. Clinical outcomes of thoracic radiotherapy for locally advanced NSCLC with EGFR mutations or EML4-ALK rearrangement. Anticancer Res 2012;32:4533-7.

18. Nakamura M, Kageyama S, Niho S, et al. Impact of EGFR mutation and ALK translocation on recurrence pattern after definitive chemoradiotherapy for inoperable stage III non-squamous non-small-cell lung cancer. Clin Lung
Cancer 2019;20:e256-e264.

19. Bauer AK, Hill T 3rd, Alexander CM. The involvement of NRF2 in lung cancer. Oxid Med Cell Longev 2013;2013:746432.

20. Jeong Y, Hoang NT, Lovejoy A, et al. Role of KEAP1/ NRF2 and TP53 Mutations in Lung Squamous Cell Carcinoma Development and Radiation Resistance. Cancer Discov 2017;7:86-101.

21. Akamatsu H, Harada H, Tokunaga S, et al. A Phase II Study of Gefitinib With Concurrent Thoracic Radiotherapy in Patients With Unresectable, Stage III Non-small-cell Lung Cancer Harboring EGFR Mutations (WJOG6911L). Clin Lung Cancer 2019;20:e25-e27.

22. Williams KJ, Telfer BA, Stratford IJ, et al. ZD1839 ('Iressa'), a specific oral epidermal growth factor receptortyrosine kinase inhibitor, potentiates radiotherapy in a human colorectal cancer xenograft model. Br J Cancer 2002;86:1157-61.

23. Ohri N, Cheng H, Jolly S, et al. The selective personalized radioimmunotherapy for locally advanced NSCLC trial (SPRINT). J Clin Oncol 2019;37:TPS8571.

24. Moding EJ, Liu Y, Nabet BY, et al. Circulating tumor DNA dynamics predict benefit from consolidation immunotherapy in locally advanced non-small-cell lung cancer. Nat Cancer 2020;1:176-83.

25. Desai S, Kim C, Veytsman I. Role of Anti-EGFR Targeted Therapies in Stage III Locally Advanced Non-small Cell Lung Cancer: Give or Not to Give? Curr Oncol Rep 2019;21:84.
Cite this article as: Singhi EK, Gay CM. Narrative review of the emerging role of molecular biomarkers in guiding the definitive management of unresectable non-small cell lung cancer. Transl Lung Cancer Res 2020;9(5):2051-2058. doi: 10.21037/tlcr-20-330 